\title{
Bases biológicas y mecánicas del tratamiento ortésico en la patología de la mano. Teoría del control de la cicatrización de Kleinert
}

\author{
R. Sánchez Rosales', M. E. Pollwein², V. J. Hernández González², C. J. Jaubert Lorenzo³, \\ R. N. Hernández-Abad González ${ }^{4}$. A. Dorta \\ 'Unidad de Cirugía de la Mano y Microcirugía, GECOT, San Cristobal de La Laguna, Tenerife. España. \\ ${ }^{2}$ Clínica de Fisioterapia NOVA, S/C de Tenerife. España. ${ }^{3}$ Fisioterapeuta Carlos Jaubert, La Palma. España. \\ ${ }^{4}$ Fisioterapia Integral S.L., San Cristóbal de La Laguna, Tenerife. España.
}

\begin{abstract}
Resumen: El propósito del presente trabajo es presentar la evolución histórica del uso del tratamiento ortésico en la patología de la mano, introducir la figura de Harold E Kleinert y su tratamiento primario de las lesiones de los tendones flexores en la zona II de la mano como principio de la "Teoría del Control de la Cicatrización" que sienta las bases biológicas y biomecánicas del tratamiento y fisioterapia postoperatoria de las patologías más frecuentes de la mano: fracturas, mano catastrófica, mano extrínseca post-traumática, mano reumática, etc.
\end{abstract}

Palabras clave: Ortesis mano, férulas dinámicas, férulas estática, tendones flexores, mano reumática, mano traumática.

\begin{abstract}
The purpose of this paper is to present the historical evolution of the hand splinting treatment, to introduce Harold Kleinert's principles for the primary repair of lacerated flexor tendons in Zone II (No man's land zone) as a base for the development of the "Theory for Controlling the Healing Process in the Hand". In this article we will show the biological and biomechanical bases of the orthotic treatment in the hand: fractures, severe hand injuries, extrinsic post traumatic hand, rheumatoid hand.
\end{abstract}

Key words: Hand splinting, hand bracing, dynamic splinting, static splinting, flexor tendons, traumatic hand splinting, rheumatoid hand.

Históricamente, el tratamiento ortésico de la mano mediante férulas comenzó en la mitad del siglo XIX!. Dos líneas paralelas emergieron en la práctica y desarrollo de férulas, una la de los cirujanos y otra la de los ortésicos. Ambos profesionales fabricaban férulas por entonces y esta dualidad se mantuvo así hasta el comienzo de 1900, con pocas colaboraciones entre ambos grupos. La gran epidemia de polio, cambiaría esta falta de colaboración. Así, cirujanos y ortésicos comenzaron a trabajar juntos durante las siguientes cuatro décadas incorporando profesionales de disciplinas emergentes como los fisioterapeutas, terapeutas ocupacionales y los médicos especialistas en medicina física, todos juntos para superar lo que constituyó la poliomielitis y sus secuelas.

Durante los años 20 y 30 del siglo pasado aparecieron los primeros cirujanos con interés en la mano en EEUU, como fue el grupo de Chicago con Kanavel al frente, siendo la única publicación al respecto un libro de Kanavel en 1912 sobre las infecciones de la mano². Parece ser que estos primeros cirujanos de mano deberían haber fabricado sus propias férulas debido a dos causas fundamentales: I) los servicios de ortesis de los hospitales estaban al 100\% dedicados a tratar las víctimas de la polio, dándose muy poca importancia a los traumatismos de la mano por entonces. 2) Los ortésicos tenían mucha experiencia en miembros inferiores, que solían ser los miembros más frecuentemente afectos en la polio, de ahí que fueran técnicamente insuficientes para proporcionar las férulas de altas demandas que requerían los cirujanos de mano. Así, durante la $2^{a}$ Guerra Mundial, tras el ataque de Pearl Harbor, la cirugía de la mano cambiaría para siempre ${ }^{3}$, debido al gran número de lesionados de la mano y el alto coste en su tratamiento, pues se necesitaba de cirujanos ortopédicos, vasculares, plásticos, etc. El presidente de los EEUU, Roosevelt, encargó a su "Surgeon General" (similar a un ministro de sanidad militar), el General Norman Kirk, crear el primer
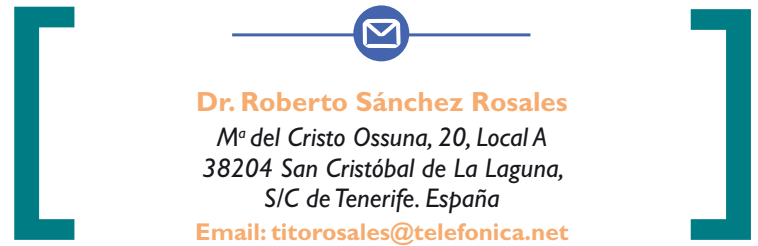
programa de formación en cirugía de la mano. Para ello eligió a un joven cirujano que ejercía en San Francisco y que ya tenía publicaciones en el campo de la cirugía de la mano, quien fue Sterling Bunnell. Así, al final de la $2^{a}$ Guerra Mundial, los cirujanos de mano eran sobradamente técnicos, con mucha experiencia, y desarrollaban todas sus férulas, pero pocos habían desarrollados sus propias férulas desde un punto de vista comercial. Incluso para el equipo apadrinado por la Casa Blanca y dirigido por Bunnell, los resultados que obtenían eran muy pobres. Así, Bunnell, en su libro de 1944, en el capítulo dedicado al tratamiento de las laceraciones de los tendones flexores exponía: "opero mis flexores y todos van bien hasta la tercera semana en donde aparece la rigidez". Esto le llevó a denominar como "The No-man's Land" (la tierra de nadie) al tratamiento de las laceraciones de los flexores en la zona II. A finales de los 50 aparece la figura de Harold Kleinert, un cirujano general de Montana que provenía de familia humilde y sin estar apadrinado por ninguna institución estatal, afincado en Louisville tras haber realizado su especialidad como cirujano general en el ejército americano. En 1967 presentó su trabajo sobre los resultados de la reparación primaria de las laceraciones de los flexores en la zona II de Bunnell, en la reunión de la ASSH (Sociedad Americana para la Cirugía de La Mano) presentando excelentes resultados en más del $88 \%$ de los casos $^{5}$. En dicho congreso, se levantó Joseph Boyes, discípulo de Bunnell y jefe de los programas de cirugía de la mano impulsados por el gobierno americano, y expuso ante toda la sociedad americana de cirugía de la mano que los resultados de Kleinert no eran creíbles. Por lo que se recomendó una auditoría de cirujanos de manos que viajó a Louisville para revisar todos los casos de Kleinert, comprobando la veracidad de los mismos ${ }^{6}$. Este hecho cambió el tratamiento de los flexores y de todas las lesiones de la mano pues fue la primera vez que se había incluido las férulas dinámicas para la movilización precoz con el objetivo de mejorar los resultados. A partir de este momento y con la introducción del microscopio quirúrgico en 19637, Kleinert realizó la primera sutura de arterias digitales constituyendo el comienzo de la microcirugía. Esto hizo necesario la incorporación de más fisioterapeutas y ortésicos en el desarrollo de nuevas férulas para el tratamiento de la patología de la mano. En 1976 se realizó el primer symposium conjunto entre cirujanos de mano, terapeutas y ortésicos, promovido por "The Philadelphia Hand Center"8 dedicado exclusivamente a la cirugía de la mano, su rehabilitación y tratamiento ortésico. Este constituyó el punto de partida en los sucesivos 30 años de numerosas publicaciones en este tema llegándose incluso a la creación del "Journal of Hand Therapy".

\section{TENDONES FLEXORES $Y$ TEORÍA DEL CONTROL DE LA CICATRIZACIÓN DE KLEINERT}

Hasta la llegada de Kleinert, no se entendía el porqué unos flexores reparados primariamente iban bien hasta que aparecía la rigidez a la segunda o tercera semana post-cirugía. Harold Kleinert pensó que si pudiera diseñar un sistema que movilizase los tendones flexores reparados sin que estos sufrieran carga alguna, las adherencias serían menores. Así el primer sistema de Kleinert para los tendones flexores estaba constituido por una férula con dos componentes: uno estático, un simple yeso que mantenía la muñeca en 30 grados de flexión y bloqueo de las articulaciones MCF (metacarpofalángicas) en 60 grados de flexión para evitar sobrecarga mecánica en los flexores reparados; y, un componente dinámico constituido por unas simples bandas de goma elástica anclados a las uñas de los dígitos a nivel distal y en el vendaje de la muñeca a nivel proximal. Así, mediante los intrínsecos y fundamentalmente los lumbricales que están proximales a las lesiones reparadas en los flexores en la zona ll, se producía la extensión de los dedos mientras que las bandas de gomas producían la flexión de los dígitos. Esto producía un desplazamiento de los flexores en el canal que impedían su adherencia9 @ Figura I.

Posteriormente, McGrouther ${ }^{10}$ publica uno de los trabajos considerados clásicos en la cirugía de la mano: "La excursión relativa de los tendones flexores en la zona II", demostrando la importancia de la flexión de las IFD (Interfalángica Distal) en la excursión o desplazamiento relativo del flexor digitorum profundis (Flexor Digitorum Profundus) con respecto al flexor digitorum superficialis (Flexor Digitorum Superficialis). Así, con cada 10 grados de flexión de la IFD se consigue un desplazamiento relativo del FDP de $1 \mathrm{~mm}$ con respecto al FDS dentro del canal. Esto llevó al equipo de Louisville a modificar su diseño inicial y añadir en el componente dinámico los tubos de rodamiento (c) Figura 2, que producían flexión de las IFDs, mejorando aún más los resultados previamente publicados".

Lo más importante es que la presunción de Kleinert de que la movilización precoz disminuía las adherencias fue posteriormente demostrado por Gelberman ${ }^{12}$ en un estudio de microscopia electrónica de barrido. Se demostró que los tendones reparados que no se 


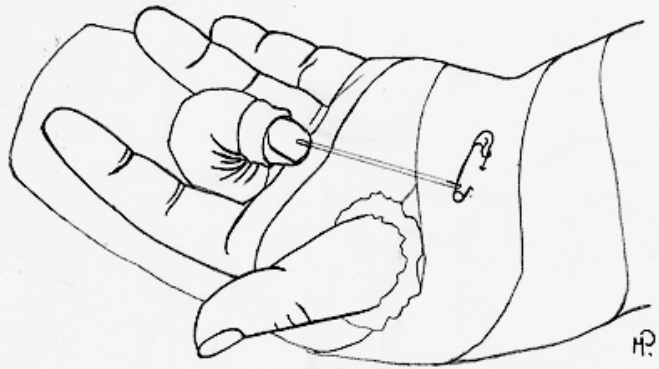

Figura I. Primer diseño de Kleinert para la movilización precoz tras reparación primaria de las laceraciones de los tendones flexores que permite la extensión activa y la flexión pasiva por medio de un elástico. Fíjese que el componente dinámico sólo produce flexión de la IFP.

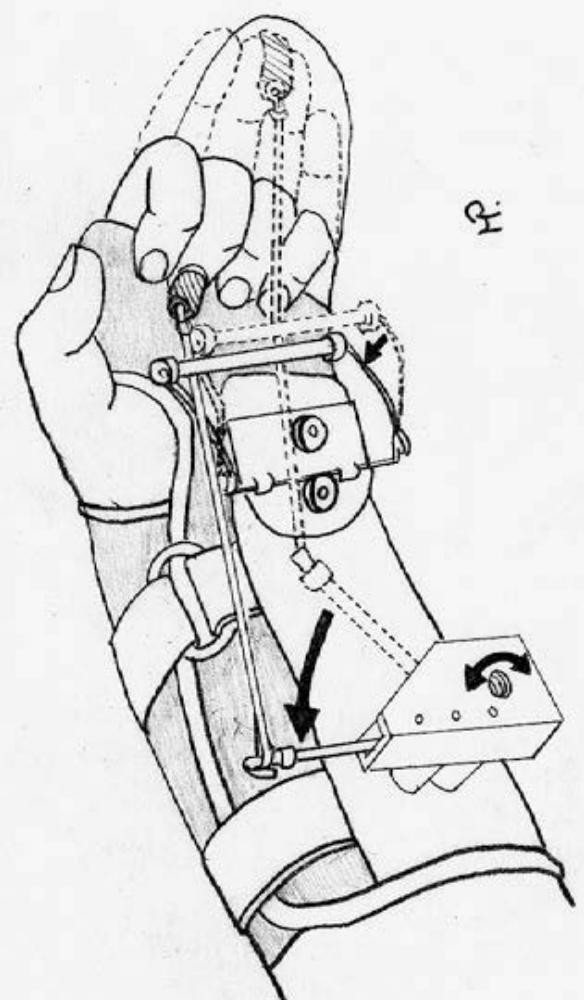

Figura 2. Férula dinámica de Kleinert del año 1988 elaborado en Louisville, E.E.U.U. Obsérvese que se añade el muelle recuperador y la barra que produce flexión de las IFDs para conseguir desplazamiento relativo entre el FDP y el FDS.

movilizaban presentaban mayor adherencia (2) Figura 3. Este hecho demostraba lo que Harold Kleinert explicaba acerca de que podíamos controlar la cicatrización de manera externa ${ }^{13}$. Así, la teoría del control de la cicatrización se basa en que biológicamente existen

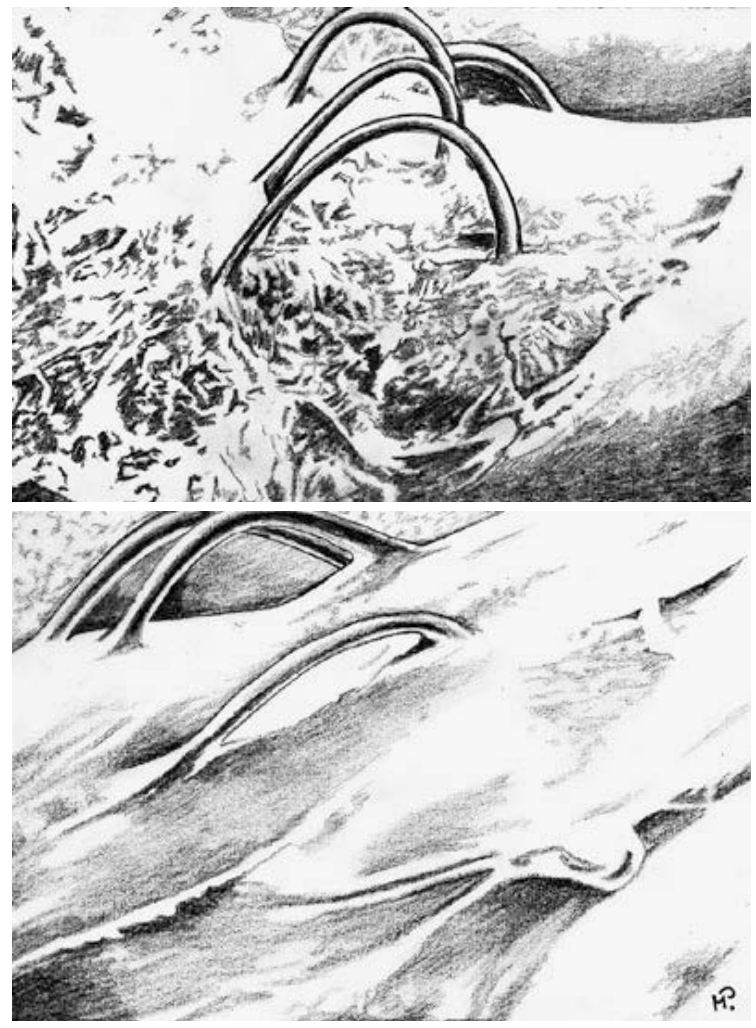

Figura 3. A. llustración basada en las imágenes publicadas por Gelberman et al 1986 ${ }^{16}$ Estudio de microscopia electrónica de barrido de un tendón no sometido a movilización precoz. Obsérvese las adherencias. B. Microscopía electrónica de barrido de un tendón sometido a movilización precoz. Obsérvese la reparación más uniforme y con menos adherencias.

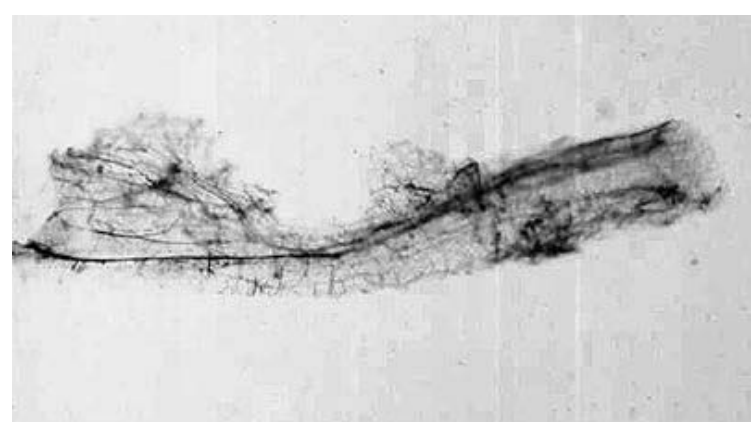

Figura 4. Microvascularización de un tendón.

en todos los tejidos del aparato locomotor un sistema vascular de pequeño tamaño denominado microvascularización'14 Figura 4. Siempre que este sistema se preserve al máximo, la reparación o cicatrización que desarrolla se denomina "restitutium ad integrum" o cicatrización primaria. Sin embargo, cuando este sistema está afectado o es insuficiente sucede la proliferación vascular procedente de los tejidos adyacentes dando lugar a la 

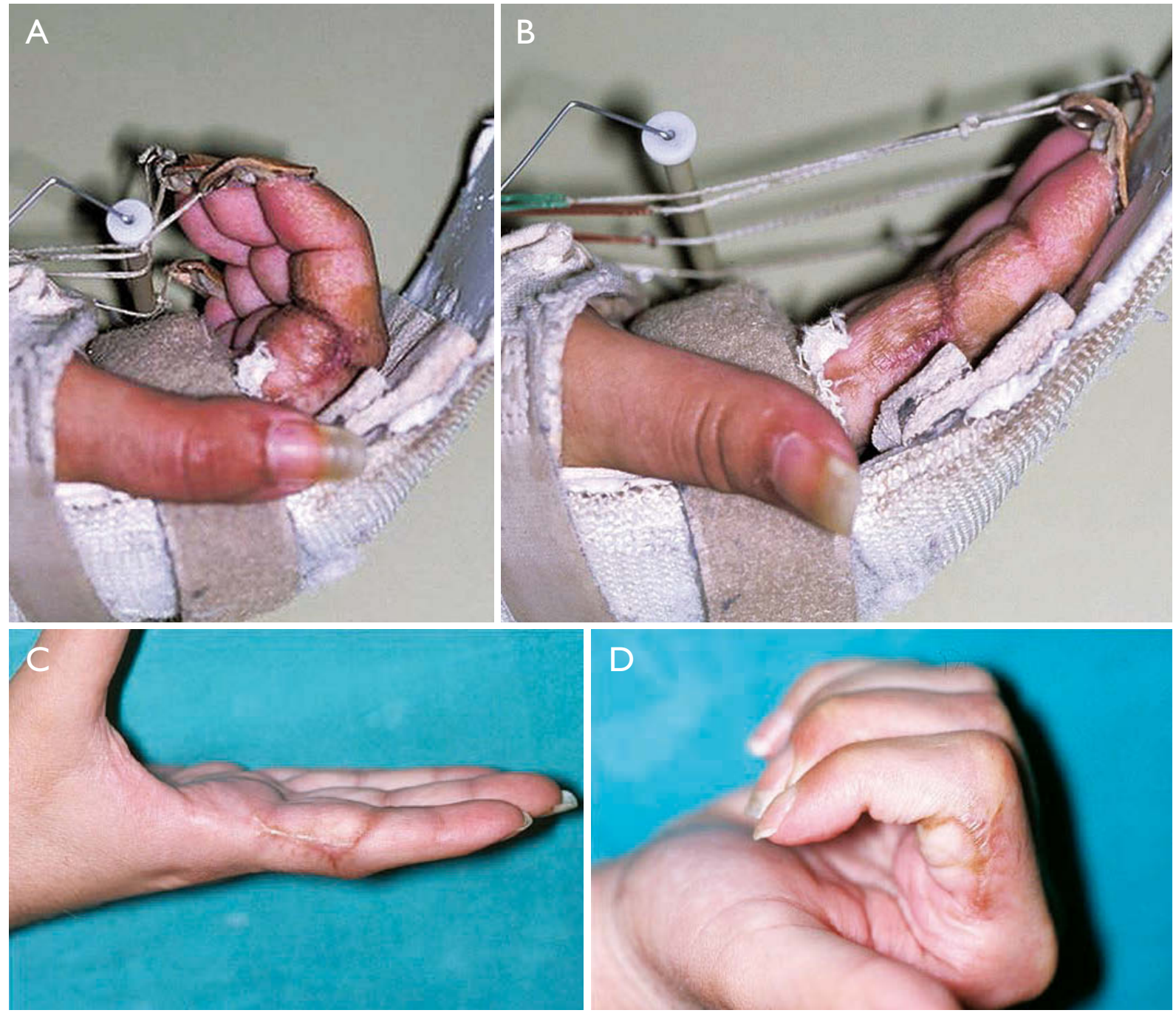

Figura 5. A-B. Sistema de Kleinert actualizado para producir flexión pasiva de las IFP y en el que el paciente realiza extensión activa de las IFs contra resistencia utilizando los intrínsecos y fundamentalmente los lumbricales. C-D. Resultado funcional a las doce semanas tras la cirugía.

reparación biológica secundaria que se realiza a través de un tejido intermedio denominado "tejido de granulación" el cual es responsable de las adherencias. En el organismo, ambas tipos de reparaciones biológicas están en equilibrio y lo que demostró Harold Kleinert es que la movilización precoz hacía inclinar la balanza biológica hacia la reparación primaria disminuyendo así las adherencias $^{13}$ @ Figura 5.

\section{APLICACIÓN DE LA TEORÍA DEL CON- TROL DE LA CICATRIZACIÓN EN LA PATOLOGÍA DE LA MANO}

Ante un traumatismo, una cirugía, un proceso inflamatorio como la artritis, etc, los tejidos de la mano van a pasar por una primera fase que dura entre cinco y diez días, caracterizado por la inflamación con aumento de la vascularización, edema, aparición de polimorfos nucleares, macrófagos, etc. Esta fase es necesaria para la reparación biológica de los tejidos, pues a partir de la semana, comienza a disminuir y aparece la fase reparativa con las células que van a cicatrizar las lesiones, manteniéndose hasta las doce semanas ${ }^{15}$.

Basados en este principio biológico, ante una lesión traumática lo primero a tener en cuenta es que tenemos dos ventanas de tratamiento. La primera llamada tratamiento primario que se puede hacer en las primeras 24 horas. Una vez se instaura el edema y el proceso inflamatorio debemos intentar disminuir ésta, hasta que la curva de la inflamación disminuya y 

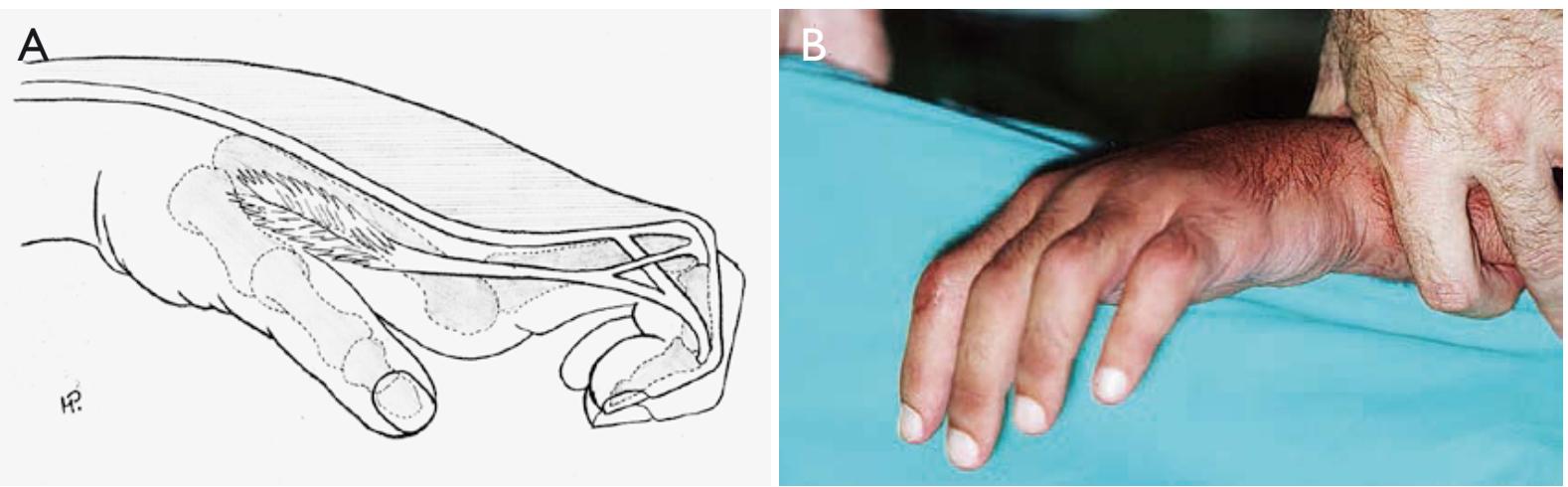

Figura 6. (A), (B). Mano extrínseca o en "garra" secundaria al edema.

poder realizar nuestro tratamiento primario diferido a la semana. Así, ante una lesión aguda de flexores el mejor resultado de la reparación primaria quirúrgica se obtiene en las primeras 24 horas. Una vez intervenido, no se instaura el tratamiento con férulas dinámicas hasta pasados al menos 4-5 días cuando la inflamación haya disminuido. El mismo principio se aplica para cualquier lesión traumática en la mano. El inicio de la inflamación con la aparición del edema no sólo debe ser disminuido, por el principio biológico antes expuesto, sino también en base a un principio biomecánico. La mano se define por el balance y equilibrio de fuerzas en cada articulación. La existencia de edema mantenido va a llevar a la mano a una situación caracterizada por imbalance de las fuerzas a favor de los extrínsecos, adoptando una posición con extensión de las MCFs y flexión de las interfalángicas por falta de función de los intrínsecos @ Figura 6. Así, en las MCFs el imbalance es a favor de los extensores extrínsecos que producen la extensión; y en las IFs (Interfalángicas), los flexores extrínsecos producen la flexión. A medida que esta posición se perpetúa en el tiempo da lugar a la rigidez debido a que los ligamentos colaterales de las MCFs están en estado de acortamiento en extensión y los ligamentos "check rain" terminan produciendo la contractura de las IFPs (Interfalángicas proximales) en flexión. A la vez, debido a la fibrina del edema y su organización fibroblástica aparece la adherencia de los extensores extrínsecos dando lugar a la "mano en garra" o "mano extrínseca".

Ante la presencia de importante edema, ya sea tras un traumatismo o un proceso inflamatorio agudo, en la que tengamos una mano extrínseca, el primer tratamiento debe ir encaminado a colocar la mano en posición funcional con una férula de descanso en una posición intrínseco-plus (-) Figura 7.
Mediante esta férula se bloquea las MCFs en flexión y las IFs en extensión. Una vez disminuido el edema en 5-7 días se pasa al tratamiento inmovilizador que corresponda. Por ejemplo: una paciente con osteoartritis primaria afectando las IFDs (enfermedad de Heberden) es intervenida quirúrgicamente mediante artrodesis de las IFDs de tres dedos largos. Tras un período de una semana, se pasó a férulas estáticas de aluminio en las IFDs. Debido a que la paciente estaba desarrollando una mano en garra extrínseca

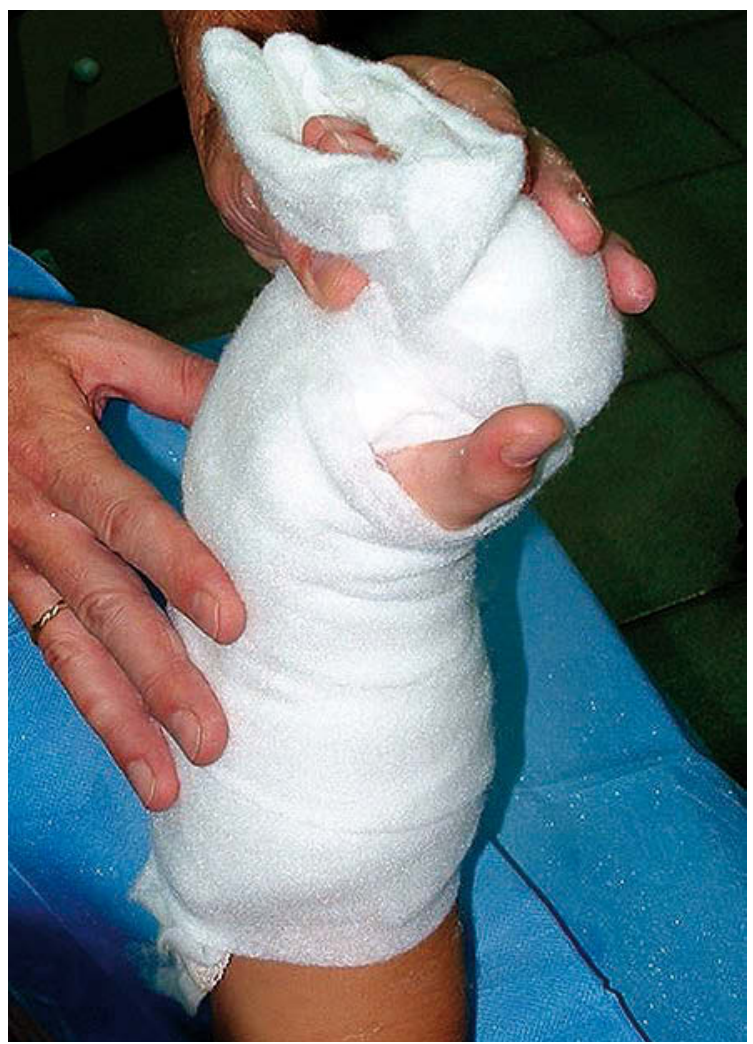

Figura 7. Férula anti-edema en posición funcional de la mano "intrínseco-plus". 

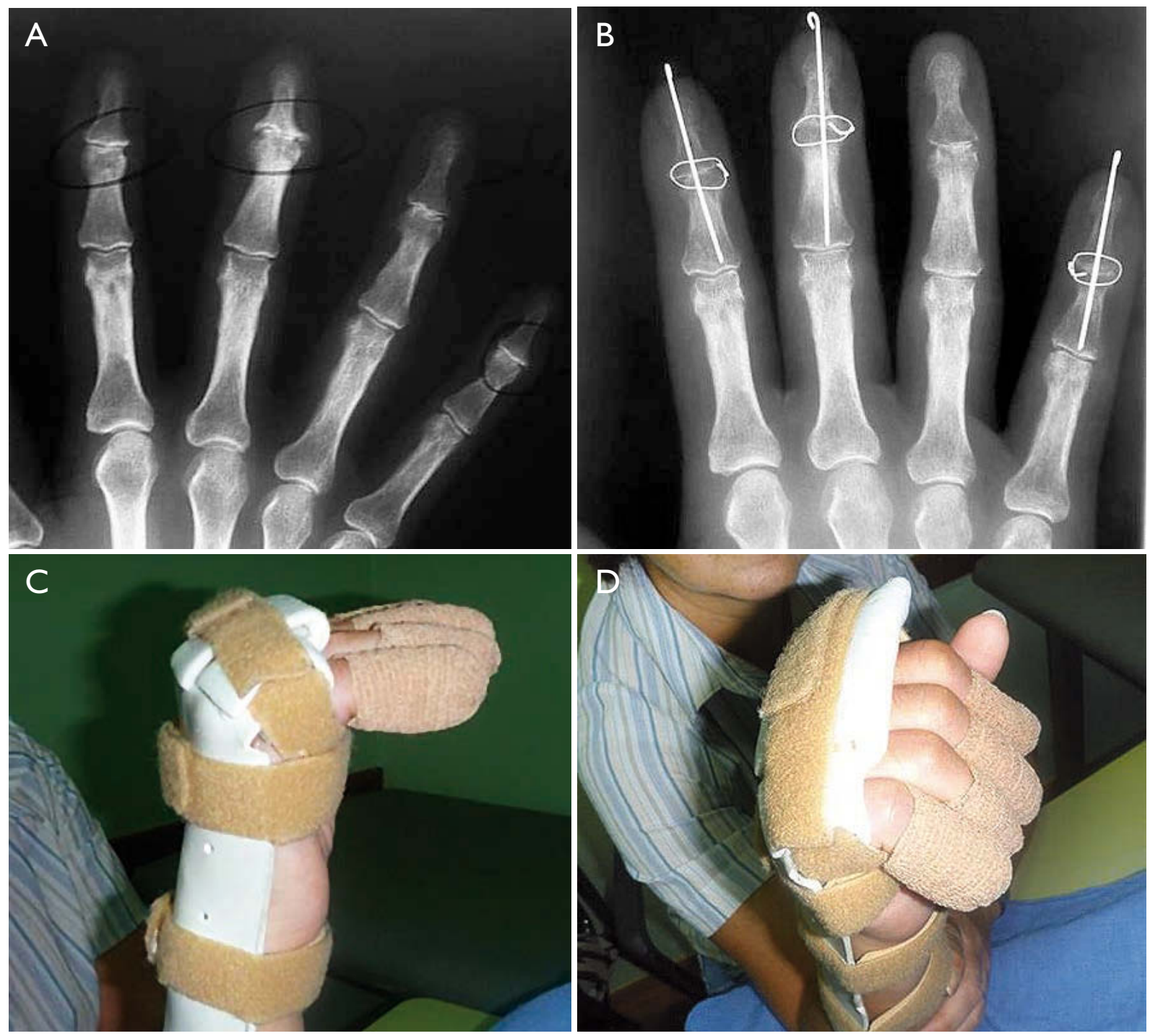

Figura 8. A. Enfermedad de Heberden en una mujer de $5 \mathrm{I}$ años, banquera, con gran discapacidad para realizar sus actividades cotidianas de la vida diaria. B. Tratamiento mediante artrodesis de las IFDs usando la técnica de aguja intramedular y cerclaje de alambre intra-óseo. C-D. Comienzo de la movilización precoz de las IFPs mientras mantenemos las MCFs en flexión debido al desarrollo de una "mano extrínseca" por edema. Las IFDs intervenidas son inmovilizadas con férulas digitales de aluminio. E-F。 Resultados.
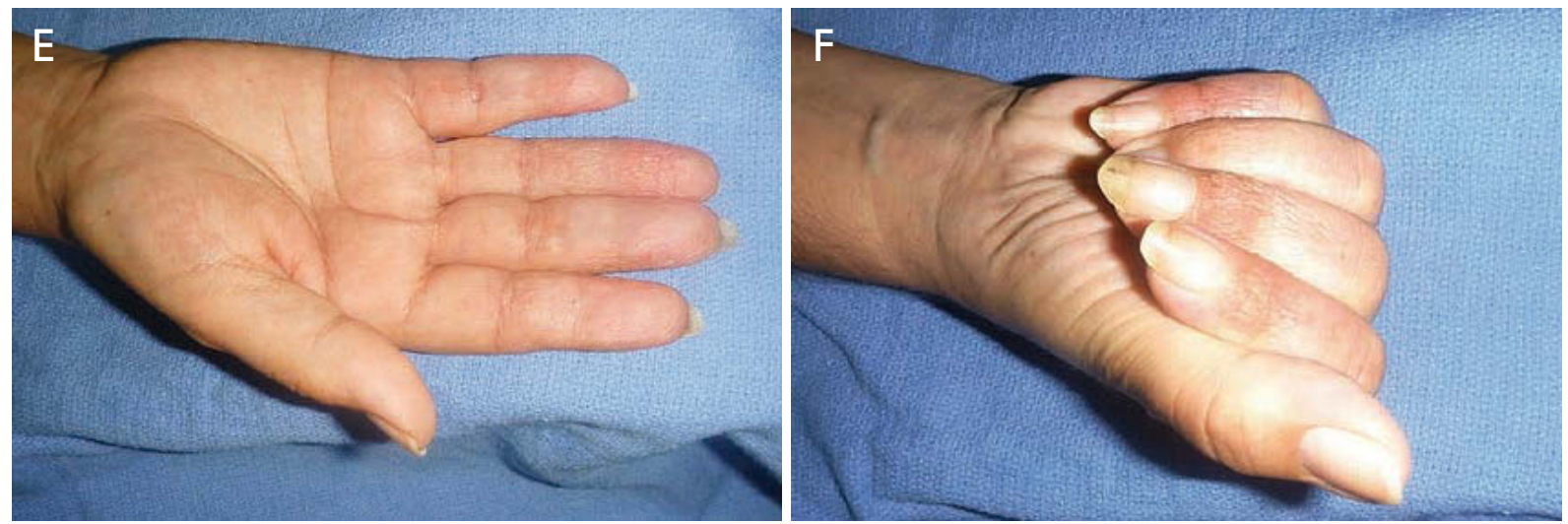


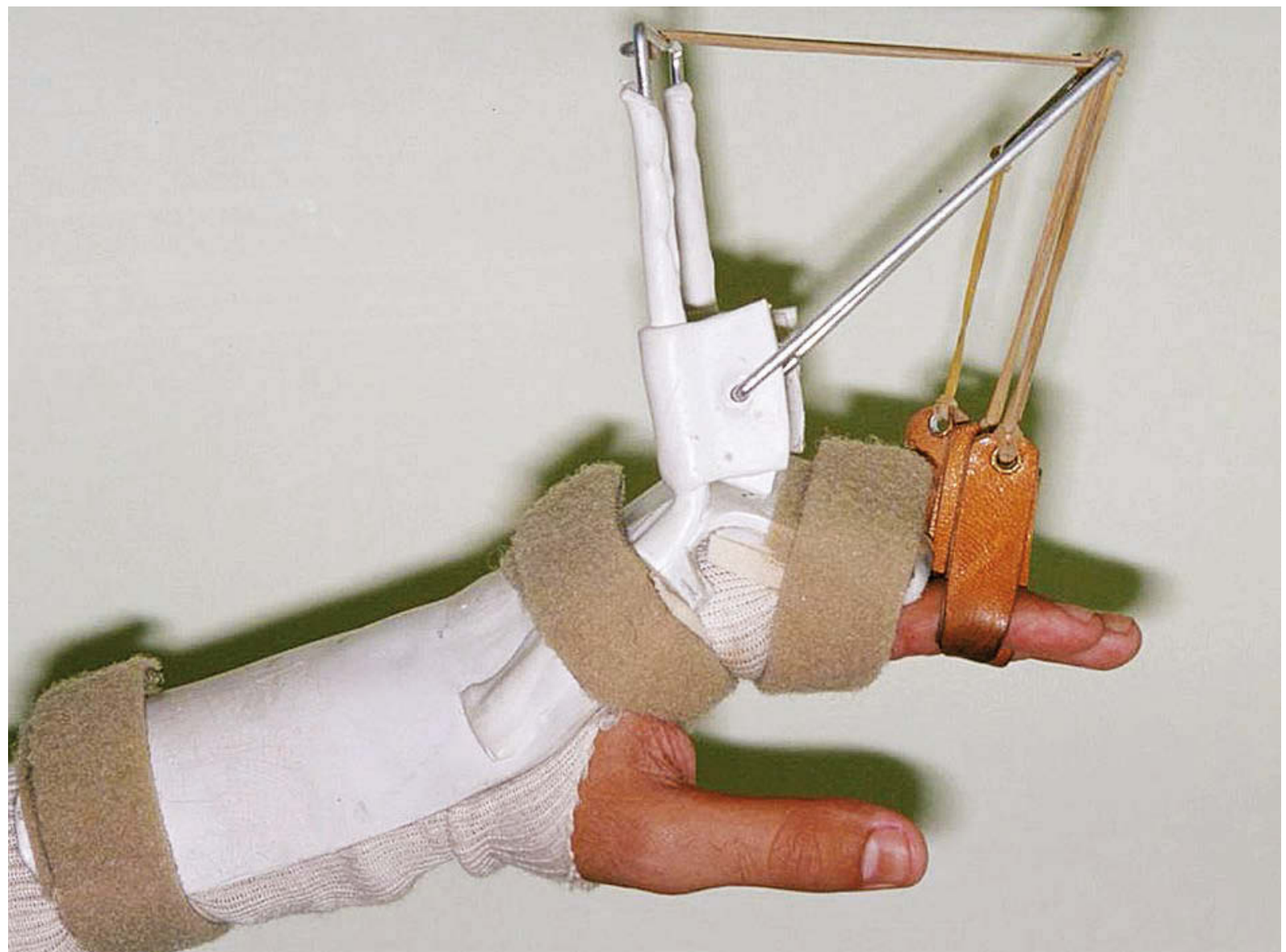

Figura 9. Férula tipo "outrigger plus crane" con bloqueo de MCFs en flexión diseñada por Kleinert para movilizar las artroplastias de las IFPs' ${ }^{16}$.

por importante edema, en este caso infrecuente, se añadió una férula de bloqueo de MCFs en flexión, para facilitar la función de los intrínsecos de manera que la paciente pueda extender las IFPs mientras las MCFs están en flexión impidiendo que aparezca la mano en garra y la rigidez (2) Figura 8.

\section{MANO CATASTRÓFICA Y TEORÍA DEL CONTROL DE LA CICATRIZACIÓN}

Aquellos traumatismos severos caracterizados por: (I) afectación tanto de los flexores como extensores extrínsecos e insuficiencia de los intrínsecos por edema, (2) en casos de reimplante y revascularización de la mano donde no va existir función de los intrínsecos por lesión de los nervios cubital y mediano; Kleinert aplicó su principio del control de la cicatrización mediante el uso de una nueva férula dinámica ${ }^{16}$ descrita inicialmente para tratar, mediante movilización precoz, las sustituciones protésicas de las IFPs, denominada "outrigger plus crane" (d) Figura 9.

Basado en los principios antes expuestos era necesario diseñar una férula dinámica que bloquease las MCFs en flexión para prevenir la extensión de las MCFs por el edema y la falta de función de los nervios cubital y mediano en los reimplantes y revascularizaciones de la mano. El sistema dinámico permitiría la extensión de las IFPs mientras que en estos casos de lesiones graves de la mano, la reparación de los flexores con suturas potentes en zona IV o V permitirían la movilización hacia la flexión de los dígitos ${ }^{17}$. Este sistema se implanta a partir del $5^{\circ}-7^{\circ}$ día tras la cirugía cuando baje el edema, permitiendo así la movilización precoz temprana, favoreciendo el máximo de cicatrización primaria y obteniendo mejores resultados (2) Figura 10. El mismo principio de las férulas dinámicas "tipo outrigger" son utilizadas incluso en las reconstrucciones diferidas del pulgar mediante transferencias de dedo de pie a mano (a) Figura II. 

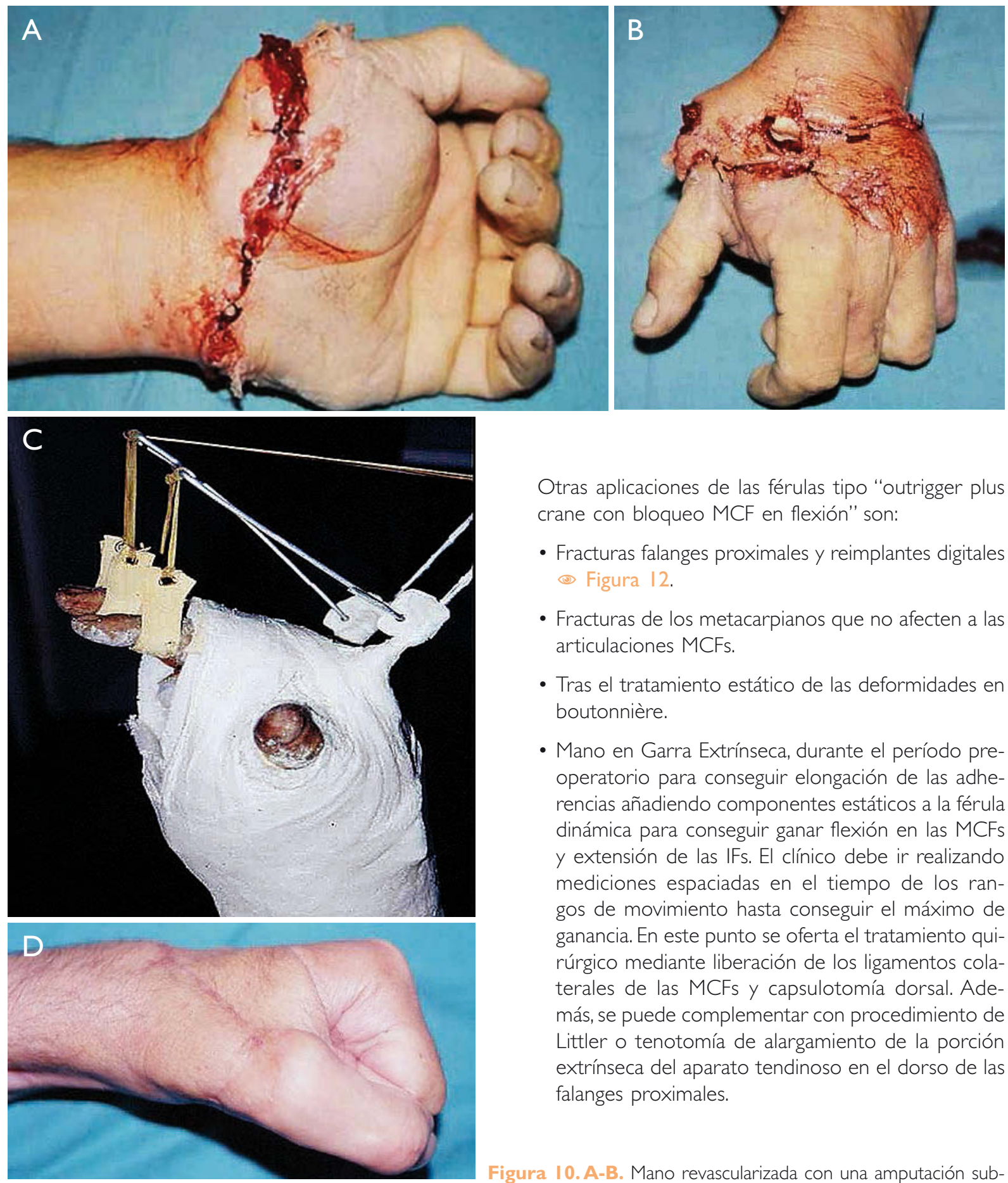

Otras aplicaciones de las férulas tipo "outrigger plus crane con bloqueo MCF en flexión" son:

- Fracturas falanges proximales y reimplantes digitales (4) Figura 12.

- Fracturas de los metacarpianos que no afecten a las articulaciones MCFs.

- Tras el tratamiento estático de las deformidades en boutonnière.

- Mano en Garra Extrínseca, durante el período preoperatorio para conseguir elongación de las adherencias añadiendo componentes estáticos a la férula dinámica para conseguir ganar flexión en las MCFs y extensión de las IFs. El clínico debe ir realizando mediciones espaciadas en el tiempo de los rangos de movimiento hasta conseguir el máximo de ganancia. En este punto se oferta el tratamiento quirúrgico mediante liberación de los ligamentos colaterales de las MCFs y capsulotomía dorsal. Además, se puede complementar con procedimiento de Littler o tenotomía de alargamiento de la porción extrínseca del aparato tendinoso en el dorso de las falanges proximales.

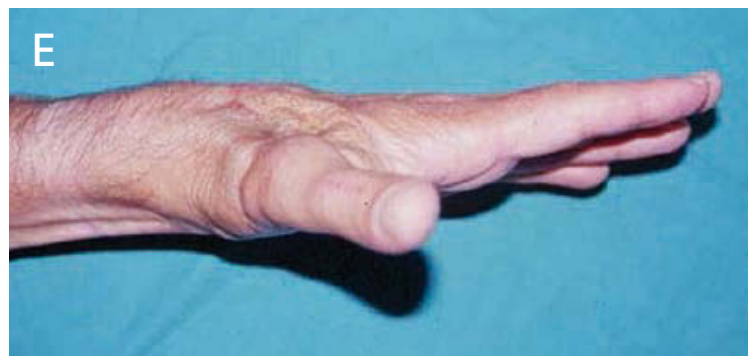

Figura \| 0. A-B. Mano revascularizada con una amputación subtotal que necesitó: osteosíntesis ósea en metacarpiano del pulgar y a nivel carpo-metacarpiana, injertos tendinosos, injerto venoso e injertos tendinosos en zona transición IV-V. C. Aplicación de la férula tipo "outrigger plus crane" en el post operatorio inmediato a los cinco días tras realizar la primera cura y haber bajado el edema. D-E. Resultado a los seis meses de la primera cirugía tras haber recibido un segundo procedimiento encaminado a la liberación del primer espacio interdigital con un colgajo cutánero-graso interóseo posterior. 


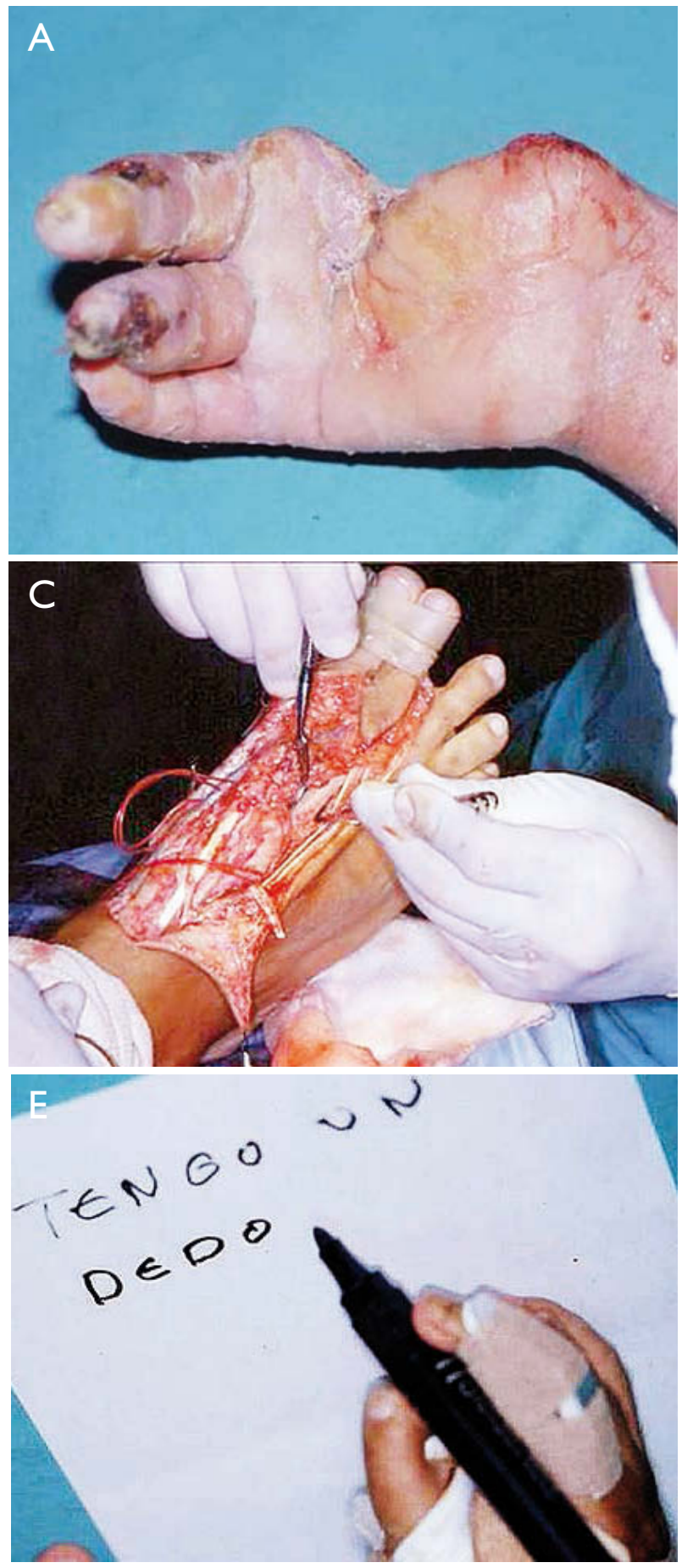

Figura II.A. Mano con amputación traumática del pulgar e índice a nivel proximal B. Cobertura cutánea en la zona de recepción de un dedo de pie a mano en un futuro tiempo quirúrgico. C. Transferencia del $2^{\circ}$ dedo del pie contralateral a la mano. D. Aplicación de la férula "tipo outrigger" en este caso para proteger los extensores extrínsecos del dedo transferido mientras que el flexor reparado con suturas tipo "tendón-loop" muy potentes permiten la movilización activa protegida. E. Resultados. El paciente puede escribir de nuevo.
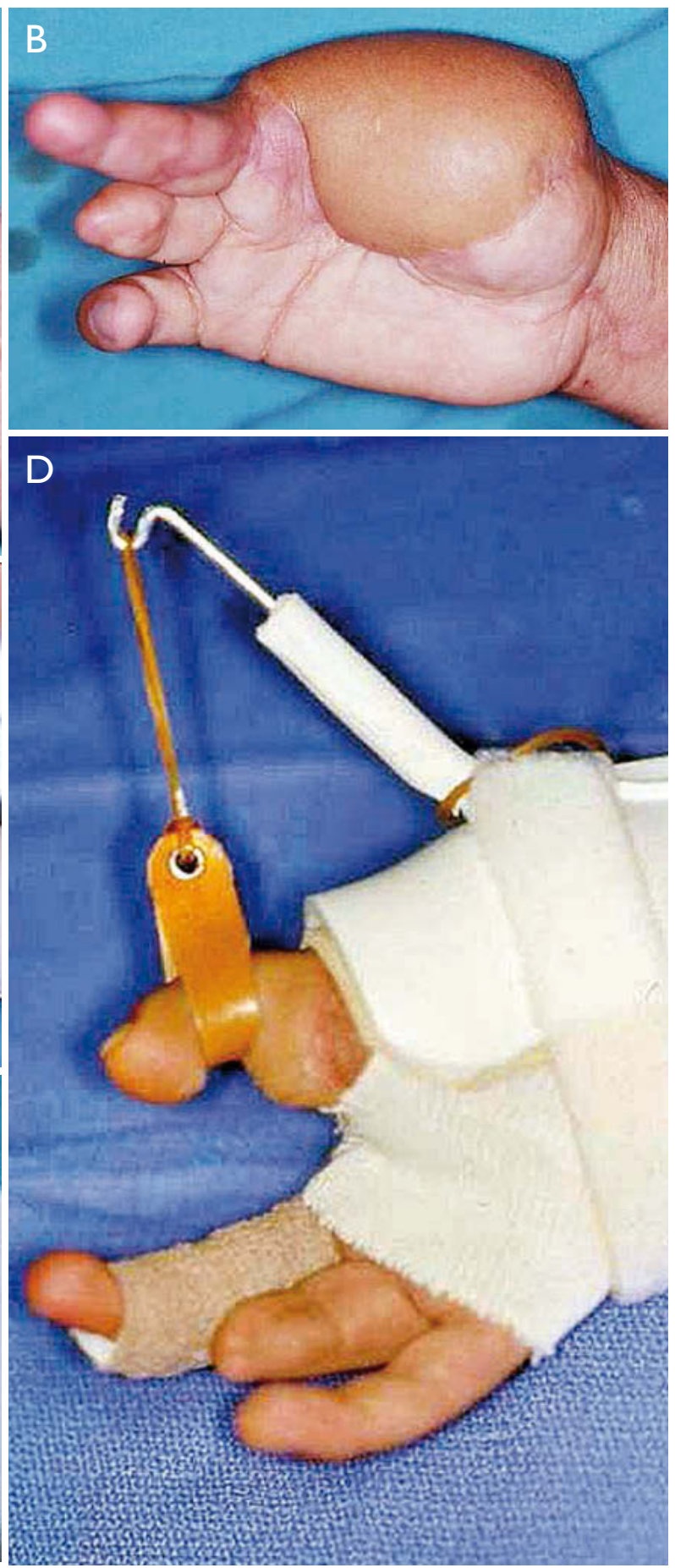

- Secuelas post-traumáticas de las IFPs, como la contractura en flexión tras esguinces, luxaciones o incluso fracturas luxaciones. También en casos de rigidez neurógena por un Síndrome Doloroso Regional Complejo Tipo I que ocasiona frecuentemente rigidez en extensión de las MCFs y rigidez de las IFs en flexión. 

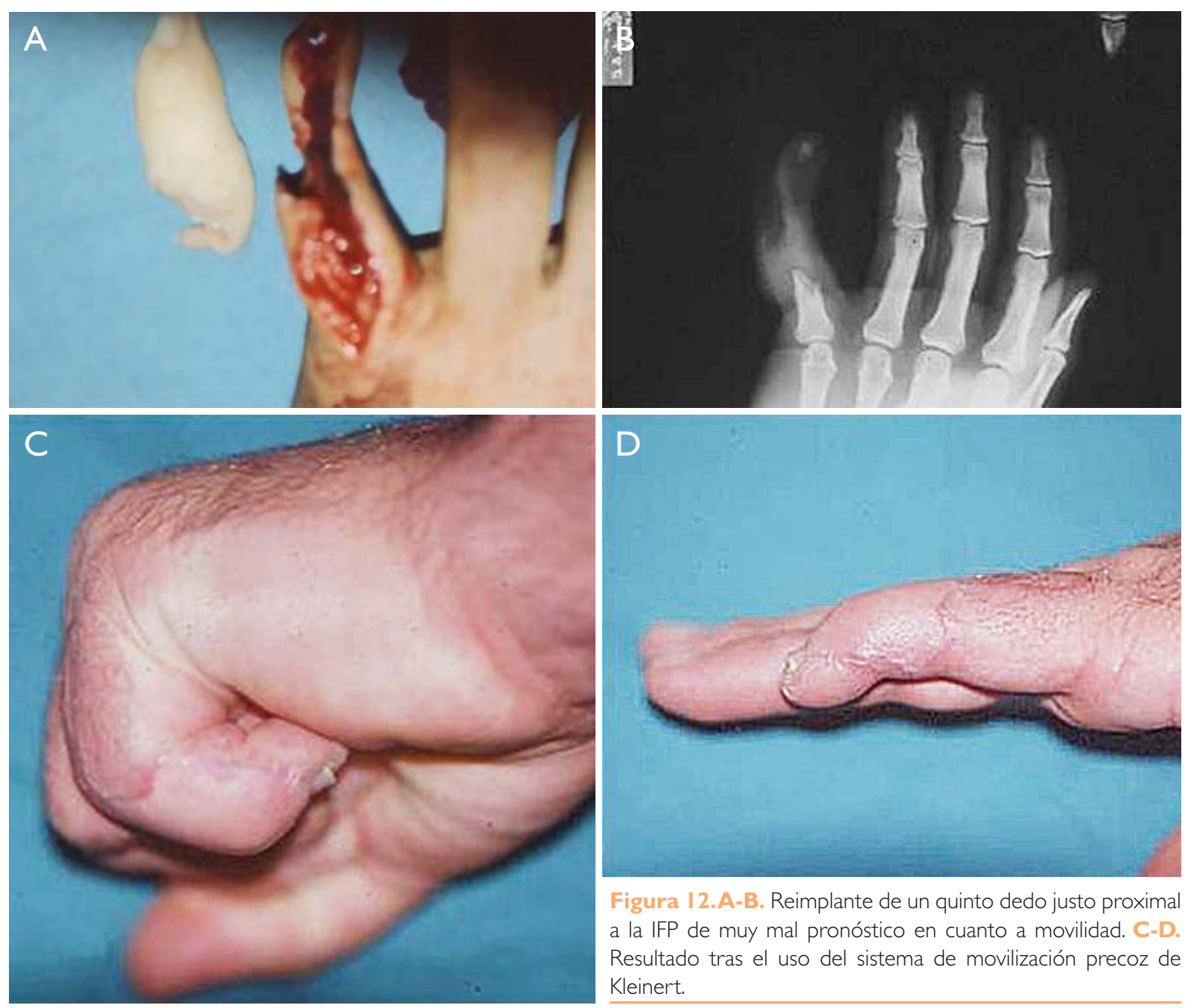

Figura I2.A-B. Reimplante de un quinto dedo justo proximal a la IFP de muy mal pronóstico en cuanto a movilidad. C-D. Resultado tras el uso del sistema de movilización precoz de Kleinert.

\section{MANO REUMÁTICA Y TEORÍA DEL CON- TROL DE LA CICATRIZACIÓN}

Hasta ahora, el principio de Kleinert de la movilización precoz dirigida para facilitar al máximo la cicatrización primaria o para modular la cicatrización secundaria existente como en los últimos casos de secuelas, se ha aplicado en una mano que biomecánicamente los extrínsecos dominaban en el balance de fuerzas. De ahí la insistencia en el componente de bloqueo de flexión de las MCFs y el intentar extender las IFs con el componente dinámico. Sin embargo, en la mano reumática, las fuerzas predominantes van a producir un desequilibrio o imbalance a favor de los intrínsecos (8) Figura I3. Estos músculos se ven contracturados, lo que lleva a la mano reumática a observar flexión excesiva de las MCFs acompañados incluso con subluxación y contractura de las placas volares. La repercusión a distal, en los dígitos, se observa con la aparición de deformidades en cuello de cisne debido a la predominancia de los intrínsecos sumado a la falta de función del FDS todo ello causado por la sinovitis y pannus.

En este tipo de mano, el tratamiento quirúrgico debe ir más allá de la simple sustitución protésica de las MCFs. Los diferentes procedimientos quirúrgicos deben estar encaminados a re-balancear la mano mediante: ( 1 ) liberación ligamentos colaterales cubitales de las MCFs dedos 2 al 5; (2) sinovectomía completa de las MCFs del 2 al 5 y sustitución protésica si fuera necesario; (3) tenotomía alargamiento de los intrínsecos cubitales del $2^{\circ}$ al $4^{\circ}$ dedos, más el Abductor Digiti Minimi evitando lesionar el tendón Flexor Digiti Minimi; (4) sinovectomía de los flexores extrínsecos sobre todo del FDS; (5) liberación de las bandas laterales en los dedos afectos de cuello de cisne; (6) re-tensado de los ligamentos colaterales radiales de las MCFs para compensar la desviación cubital y (7) 


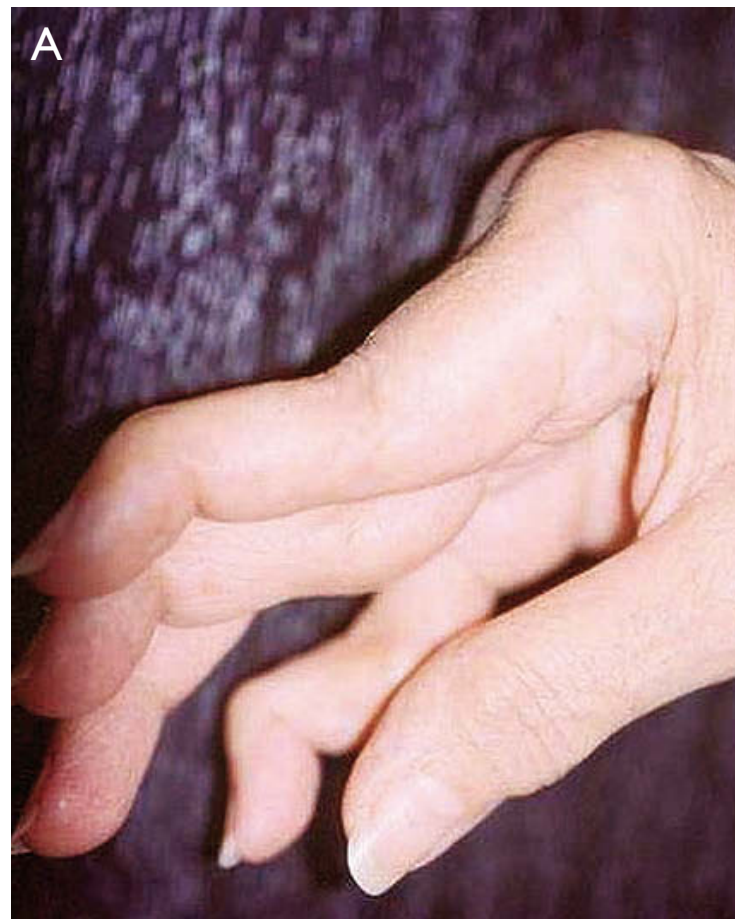

recentralización de tendones extensores extrínsecos tras el cierre de las MCFs. Sin embargo, lo más importante es otra vez el tratamiento ortésico postoperatorio. Tras bajar el edema con un vendaje de confort, al $5^{\circ}-7^{\circ}$ día postoperatorio, se pasa a una férula dinámica que a diferencia de todas las vistas hasta ahora lo que se intenta es poner la mano en una posición extrínseca con MCFs en extensión y las IFs en flexión. De esta manera los intrínsecos cicatrizaran en su máxima longitud, desarrollándose la flexión activa de las MCFs mediante los intrínsecos radiales que no fueron tenotomizados. Esta férula dinámica "tipo outrigger" en donde no existe bloqueo en flexión de las MCFs, las bandas de tracción inciden en las falanges proximales de forma oblicua. Añadiendo un segundo componente de tracción lateral sobre el $2^{\circ}$ y $3^{\text {er }}$ dedo para tener tres ejes de tracción. Así se permite: primero, evitar la desviación cubital; segundo, facilitar la supinación de los dígitos 2 y 3 para la pinza tridigital. Inicialmente, el material no es de termoplástico sino de yeso debido al postoperatorio inmediato. Una vez llegamos a los quince días, se retiran puntos y se pasa a la férula dinámica tipo termoplástico que llevará hasta las doce semanas (d) Figura 14.

\section{CONCLUSIONES}

Hemos visto cómo un principio básico obtenido del tratamiento de los flexores en la zona II se fue apli-

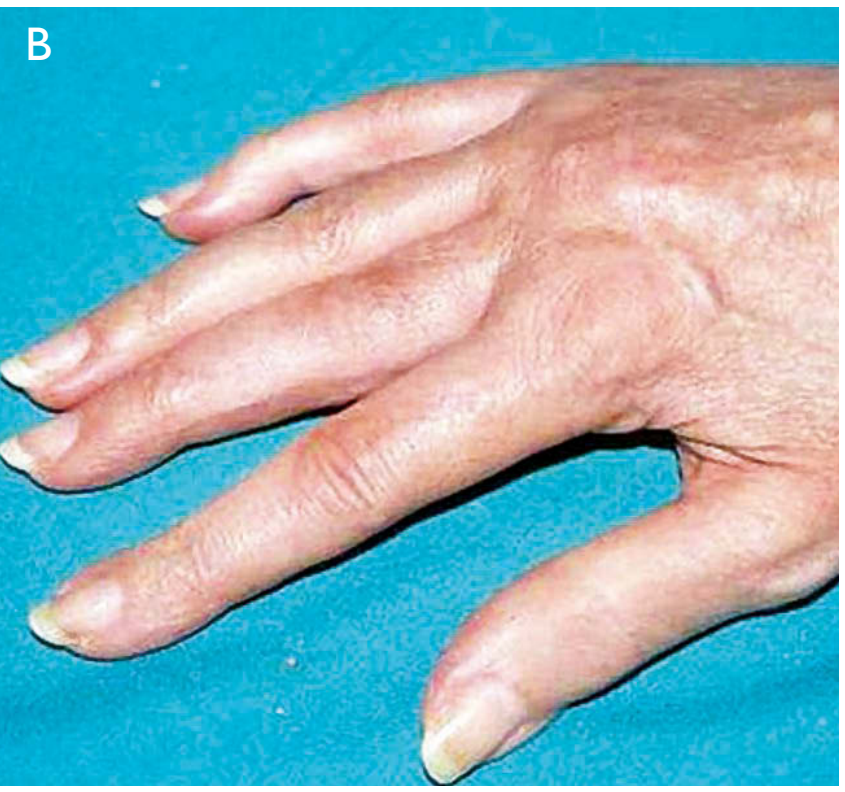

Figura | 3.A. Mano reumática de predominio intrínseco con flexión y subluxación volar de las MCFs, desviación ulnar y deformidades en cuello de cisne. B. Resultado tras reconstrucción. cando a diferentes patologías con diferentes férulas y basado en dos principios que definen la teoría del control de la cicatrización de Kleinert: intentar balancear las fuerzas mecánicas en la mano y aplicar movimiento precoz protegido para obtener el máximo que se pueda de cicatrización primaria posible para evitar la rigidez. Estos principios se siguen manteniendo

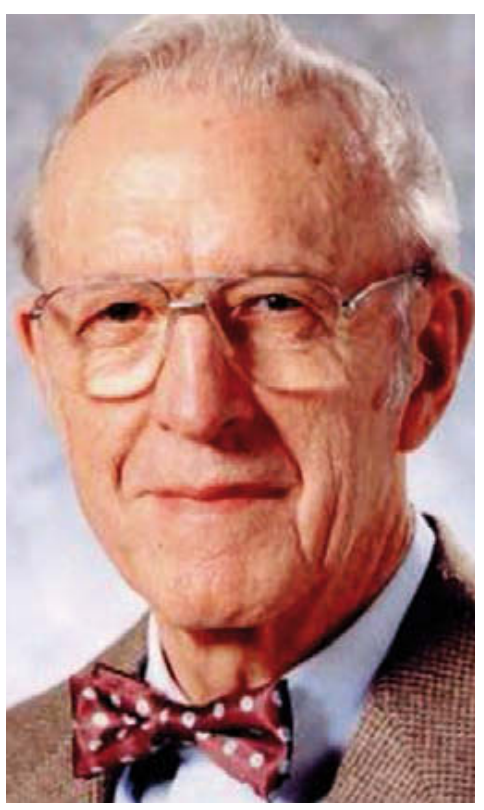

Harold E Kleinert (1921-2013) 


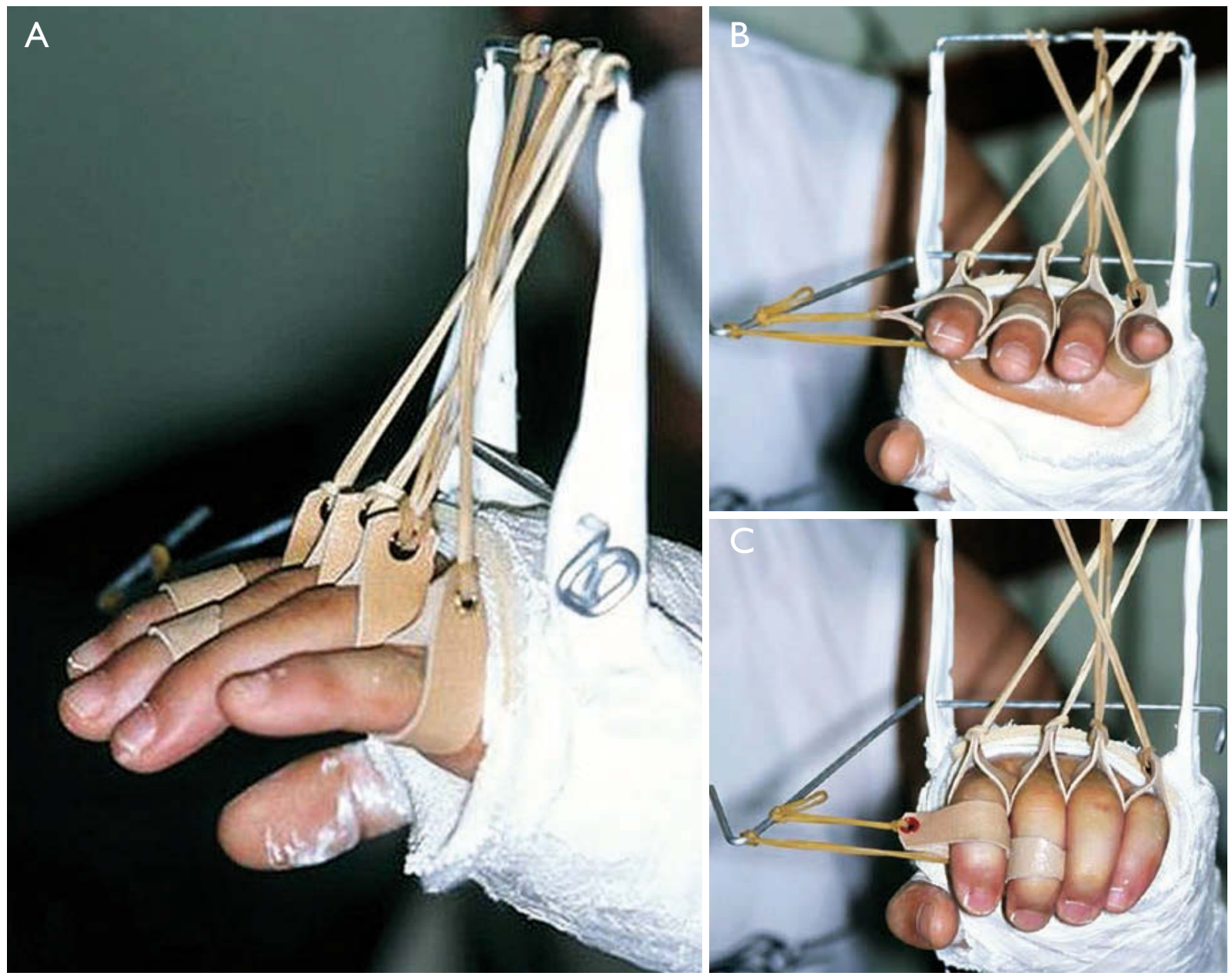

Figura |4.A. Aplicación del sistema "outrigger" en una mano reumática tras cirugía una vez pasado el tiempo inicial de 5 a 7 días. Obsérvese la posición en mano extrínseca con tracción de las falanges proximales que las lleva a extensión. B. Obsérvese las tracciones que son oblicuas siendo desde cubital para los dedos 2 y 3, mientras que las tracciones son más radiales para los dedos 4 y 5. Las tracciones laterales a los dedos 2 y 3 junto con las fuerzas de flexión favorecen la supinación a los dedos radiales. C. Flexión activa tanto de MCFs como de IFs.

hasta nuestros días en la mayoría de la patología de la mano 6, 18, 19,20. De lo cual se deduce que la rehabilitación es tan importante como la cirugía. Siendo fundamental una buena comunicación entre cirujano y terapeuta de la mano para que el procedimiento rehabilitador suceda de forma correcta en el tiempo.

\section{CONFLICTOS DE INTERESES}

Los autores declaran no tener conflictos de intereses.

\section{BIBLIOGRAFÍA}

I. Ernst FG. Orthopaedic Apparatus. London, UK: Sprague; 1883.
2. Kanavel A. Infections of the Hand. Philadelphia and New York: Lea \& Febiger; 1912.

3. Carter P. The Embryogenesis of the specialty of hand surgery: a story of three great americans - a politician, a general, and a duck hunter: The 2002 Richard J. Smith Memorial Lecture. J Hand Surg. 2002;28A: I 85-98.

4. Bunnell S. Surgery of The Hand (first edition). Philadelphia: Lippincott; 1944.

5. Kleinert HE, Kutz JE, Ashbell S, Martinez E. Primary repair of flexor tendons in no-man's land. J Bone Joint Surg Am. 1967:49A:577.

6. Newmeyer WL, Manske PR. No man's land revisited: the primary flexor tendon repair controversy. J Hand Surg Am. 2004;29: I-5. 
7. Kleinert HE, Kasdan ML, Romero JL. Small blood vessel anastomosis for salvage of severely injured upper extremity. J Bone Joint Surg Am. 1963;45A:788-96.

8. Fess EE. A History of splinting: To Understand the present, view the past. J Hand Therapy. 2002; 1 5:97132.

9. Kleinert HE, Kutz J, Atasoy E, Stormo A. Primary repair of flexor tendons. Orthop Clin North Am. 1973:4:865-76.

10. McGrouther DA,Ahmed MR. Flexor tendon excursions in "no-man's land". Hand. 1981;13:129-41.

I I. Lister GD, Kleinert HE, Kutz JE, Atasoy E. Primary flexor tendon repair followed by immediate controlled mobilization. J Hand Surg Am. 1977;2: $44|-5|$.

12. Gelberman RH, Manske PR, Akeson WH, Woo SL, Lundborg G, Amiel D. Flexor tendon repair. J Orthop Res. 1986;4:1 19-28.

13. Kleinert HE, Cash SL. Management of acute flexor tendon injuries in the hand. Instr Course Lect. 1985;34:36 |-72.

14. Rosales RS. Valoración crítica de los medios de infusión en el estudio de la microvascularización del Aparato Locomotor. Tesis Doctoral. Universidad La Laguna. Tenerife. 1992.
15. Burke FD, McGrouther DA, Smith PJ. Principles of Hand Surgery. London: Churchill Livingstone; 1990.

16. Chesher SP, Schwartz KS, Kleinert HE. A new early mobilization splint for PIP joint replacement. J Hand Therapy. 1988; I:200-3.

17. Scheker LR, Chesher SP, Netscher DT, Julliard KN, $O$ N Neill WL. Functional results of dynamic splinting after transmetacarpal, wrist, and distal forearm replantation. J Hand Surg Br. 1995;20:584-90.

18. Jabłecki J, Syrko M, Arendarska-Maj A. Rehabilitation of fingers after flexor tendon lesions qualified for secondary reconstruction, using Kleinert's dynamic splinting method. Ortop Traumatol Rehabil. 2006;31:517-21.

19. Starr HM, Snoddy M, Hammond KE, Seiler JG $3 r d$. Flexor tendon repair rehabilitation protocols: a systematic review. J Hand Surg Am. 2013;38: 17|2-7.

20. Sameem M, Wood T, Ignacy T, Thoma A, Strumas N. A systematic review of rehabilitation protocols after surgical repair of the extensor tendons in zones V-VIII of the hand. J Hand Ther. 20 I I; 24:365-72. 